\title{
INTEGRAL CAYLEY SUM GRAPHS AND GROUPS
}

\author{
XUANLONG MA ${ }^{1,2}$ AND KAISHUn WANG ${ }^{2}$ \\ ${ }^{1}$ College of Mathematics and Information Science \\ Guangxi University, Nanning 530004, China \\ ${ }^{2}$ Sch. Math. Sci. \& Lab. Math. Com. Sys. \\ Beijing Normal University \\ Beijing 100875, China \\ e-mail: xuanlma@mail.bnu.edu.cn \\ wangks@bnu.edu.cn
}

\begin{abstract}
For any positive integer $k$, let $\mathcal{A}_{k}$ denote the set of finite abelian groups $G$ such that for any subgroup $H$ of $G$ all Cayley sum graphs $\operatorname{CayS}(H, S)$ are integral if $|S|=k$. A finite abelian group $G$ is called Cayley sum integral if for any subgroup $H$ of $G$ all Cayley sum graphs on $H$ are integral. In this paper, the classes $\mathcal{A}_{2}$ and $\mathcal{A}_{3}$ are classified. As an application, we determine all finite Cayley sum integral groups.
\end{abstract}

Keywords: Cayley sum graph, integral graph, Cayley sum integral group.

2010 Mathematics Subject Classification: 05C25, 05C50.

\section{REFERENCES}

[1] A. Abdollahi and M. Jazaeri, Groups all of whose undirected Cayley graphs are integral, European J. Combin. 38 (2014) 102-109. doi:10.1016/j.ejc.2013.11.007

[2] A. Abdollahi and E. Vatandoost, Which Cayley graphs are integral?, Electron. J. Combin. 16 (2009) \#R122.

[3] A. Ahmady, J.P. Bell and B. Mohar, Integral Cayley graphs and groups, SIAM J. Discrete Math. 28 (2014) 685-701. doi:10.1137/130925487

[4] N. Alon, Large sets in finite fields are sumsets, J. Number Theory 126 (2007) $110-118$. doi:10.1016/j.jnt.2006.11.007 
[5] M. Amooshahi and B. Taeri, On Cayley sum graphs of non-Abelian groups, Graphs Combin. 32 (2016) 17-29. doi:10.1007/s00373-015-1535-4

[6] A.E. Brouwer and W.H. Haemers, Spectra of Graphs (Springer-Verlag, New York, 2012). doi:10.1007/978-1-4614-1939-6

[7] F.C. Bussemaker and D.M. Cvetković, There are exactly 13 connected, cubic, integral graphs, Univ. Beograd. Publ. Elektrotehn. Fak. Ser. Mat. Fiz. 544-576 (1976) $43-48$.

[8] B. Cheyne, V. Gupta and C. Wheeler, Hamilton cycles in addition graphs, RoseHulman Undergrad. Math J. 4 (2003) 1-17.

[9] F.R.K. Chung, Diameters and eigenvalues, J. Amer. Math. Soc. 2 (1989) 187-196. doi:10.1090/S0894-0347-1989-0965008-X

[10] M. DeVos, L. Goddyn, B. Mohar and R. Sámal, Cayley sum graphs and eigenvalues of (3,6)-fullerenes, J. Combin. Theory Ser. B 99 (2009) 358-369. doi:10.1016/j.jctb.2008.08.005

[11] I. Estélyi and I. Kovács, On groups all of whose undirected Cayley graphs of bounded valency are integral, Electron. J. Combin. 21 (2014) \#P4.45.

[12] B.J. Green, Counting sets with small sumset, and the clique number of random Cayley graphs, Combinatorica 25 (2005) 307-326. doi:s00493-005-0018-2

[13] F. Harary and A.J. Schwenk, Which graphs have integral spectra? in: R.A. Bari and F. Harary (Eds.), Graphs and Combinatorics, Lecture Notes in Math. 406 (Springer, 1974) 45-51. doi:10.1007/bfb0066434

[14] W. Klotz and T. Sander, Integral Cayley graphs over abelian groups, Electron. J. Combin. 17 (2010) \#R81.

[15] X. Ma and K. Wang, On finite groups all of whose cubic Cayley graphs are integral, J. Algebra Appl. 15 (2016) 1650105, 10 pp. doi:10.1142/S021949881650105X

[16] A.J. Schwenk, Exactly thirteen connected cubic graphs have integral spectra, in: Y. Alavi and D.R. Lick (Eds.), Theory and Applications of Graphs, Lecture Notes in Math. 642 (Springer, 1978) 516-533. doi:10.1007/BFb0070407

[17] W. So, Integral circulant graphs, Discrete Math. 306 (2006) 153-158. doi:10.1016/j.disc.2005.11.006

Received 6 January 2015

Revised 12 October 2015

Accepted 27 November 2015 